\title{
A Tandem Queueing Model for Delay Analysis in Disconnected Ad Hoc Networks
}

\author{
Ahmad Al-Hanbali, Roland de Haan, Richard J. Boucherie, and Jan-Kees van Ommeren \\ University of Twente, Enschede, The Netherlands
}

\section{ABSTRACT}

Ad hoc network routing protocols may fail to operate in the absence of an end-to-end connection from source to destiJ nation. This deficiency can be resolved by so-called opportunistic networking which exploits the mobility of the nodes by letting them operate as relays according to the store3 carry-and-forward paradigm. However, the efficiency of this 3 approach will depend to a large extent on the contact and .. inter-contact times of node pairs.

E-

In this work, we analyze the delay performance of a small opj portunistic network by considering a tandem queueing syso tem. We present an exact packet-level analysis by applying ideas from the polling literature. Due to the state-space Un expansion, this analysis cannot efficiently be applied for all

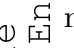
model parameter settings. For this reason, an analytical approximation is constructed and its excellent performance has (j) extensively been validated. Numerical results on the mean + end-to-end delay show that the inter-contact time distribu(1) tion impacts this metric only through its first two moments. Finally, we study delay optimization under power control.
Keywords: Tandem model, Delay-tolerant networking, Op-

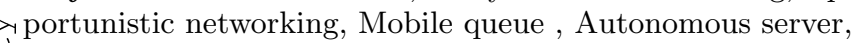
각 Performance analysis.

\section{INTRODUCTION}

End-to-end connectivity is not a natural property of ad hoc networks. For instance, nodes may vary their transmission power, nodes may move, nodes may enter the sleep mode, or nodes may suffer from hardware failures. As a result, the network structure changes dynamically and this may lead to undesired situations of nodes becoming disconnected from parts of the network.

The traditional store-and-forward routing protocols, which require the existence of a connected path between a source and a destination, cannot be employed in highly disconnected ad hoc networks. A solution for this problem is to exploit the mobility of nodes present in the network. The mobile nodes may form in fact bridges which relay traffic between the disconnected parts. This approach has been proposed in the pioneering paper of Grossglauser and Tse [14] as an alternative to the store-and-forward paradigm and it is now known as the store-carry-and-forward paradigm in the context of delay-tolerant networking (DTN) [1]. In DTN, the incurred delay to send data between nodes can be very large and unpredictable due to the disconnection problem. Applications of such can be found in, e.g., disaster relief networks, rural networking, environmental monitoring networks, vehicular networks, and interplanetary networks.

A significant amount of research for routing-based approaches in DTN has recently emerged. An important factor in DTN is the so-called contact opportunity between node pairs. Two nodes are in contact if they are within transmission range of one another and thus packet exchange between them is possible. The proposed routing solutions essentially differ on the required knowledge of these contact opportunities. Specifically, depending on whether the contact opportunities are scheduled [16], predicted [20], controlled [33], or opportunistic $[25,27]$, they can be grouped into different classes. The best performance would be achieved in the full knowledge case of contacts. However, this comes at the expense of a higher complexity both from the implementation and from the maintenance perspectives. In the present work, we will focus on the performance analysis of the opportunistic-based approach where no knowledge is required. For detailed surveys about the different routingbased approaches in DTN we refer to [28, 29].

Another factor that impacts the performance of opportunistic approaches is the inter-contact time which is defined as the time duration between two consecutive contacts of node 
pairs. The inter-contact time mainly depends on the mobility of the nodes. In [13], simulations showed that for the Random Waypoint [2] and the Random Direction [23] mobility models the distribution of the inter-contact times is exponential when the nodes' transmission range is small. On the contrary, for human mobility, it is shown through experiments that the tail of the distribution of inter-contact times has a power law decay in some finite range [4], and after that it exhibits an exponential decay [17]. In the present work, we will assume that the inter-contact times distribution has a finite first and second moment.

We will analyze the opportunistic approaches in DTN by taking into account, unlike $[4,13,26]$, that the transmission of packets may fail due to the short contact time and a retransmission is required. Also, we assume that the source node has a stream of packet arrivals instead of only one packet, like it was considered in $[15,26,32]$. In addition, here we are interested in what happens in a more practical case of small, finite-size networks, rather than in asymptotic cases (see, e.g., $[14,32]$ ). To this end, we adopt the network scenario of a fixed source and destination node and mobile intermediate nodes that serve as relay nodes. As a primary step towards understanding such models, we will study a network model with a single mobile node as a relaying device. Although it is a small model, it contains the main characteristics of an opportunistic network and it is also non-trivial from an analytical perspective.

The network model of our interest is reminiscent of a twoqueue tandem model with a single alternating server. Such a tandem model has been analyzed under various servicing strategies (see, e.g., [?]). Typically, these strategies are based on the assumption that the server can be controlled. However, in the mobility-driven model of our interest, the server is autonomous and there is no possibility to control its movement. The research efforts on models with timelimited service periods are also closely related to our work. In a two-queue setting, [5] analyzes the model via boundary value techniques. Unfortunately, the analysis along these lines for more than two queues appears intractable. Timelimited service models have also been studied in the context of polling systems (see, e.g., $[10,22]$ ). However, also in these models, there exists a notion of server control, since it is assumed that whenever a queue becomes empty the server moves to another queue.

In this work, our interest is mainly in the end-to-end delay under opportunistic networking. We assume that data packets arrive according to a Poisson process at the source queue. The mobile node stores the packets received from the source and forwards them to the destination. The source and destination are assumed far apart, so that the mobile node is never in range of both source and destination. The contact times are assumed exponentially distributed. Packets whose transmission is interrupted will be retransmitted during a next contact time.

We study this system at the packet level by considering the tandem queueing model as a particular kind of polling system. That is, a polling system for which customers of one queue move to another queue after being served. This specific polling system is a time-limited polling system extended with the feature that the server remains at a queue even if it becomes empty. We perform an exact analysis for this system by using similar techniques as in [22] and [6].

Due to the state-space expansion, the computation time of the joint queue-length probabilities may grow large for certain model parameters. Therefore, as a complementary tool, we present an analytical approximation for the case that the service requirements at each queue are exponential. The queue-length process at the second queue is then analyzed in isolation as a workload process with Poisson batch arrivals. The Poisson process follows directly from the assumption of exponential contact times. The key element is to approximate the batch size distribution as closely as possible. A similar model has been analyzed by Borst et al. [3]. The authors consider a Poisson batch arrival process for which the batch size depends on the inter-arrival time of the batch. This differs from our model in the sense that batch sizes depend not only on the final inter-arrival period, but also on the previous ones which induce that they are dependent.

Numerical experiments show the excellent performance of the approximation for a broad range of parameter settings. These experiments further show that mean sojourn time is insensitive to third and higher moments of the inter-contact times. Finally, several guidelines are given for delay optimization by power control. In particular, balancing the queues load is not always close to the optimal policy. However, using a simple heuristic based on optimizing the delay of a tandem model of two M/M/1 queues gives nearly optimal results under wide variety of parameter settings.

The main contributions of this article are:

- an analytical model for queue-length and delay analysis in a simple opportunistic network;

- an analytic approximation for the delay in a two-queue tandem model with a single autonomous server;

- third and higher moments of the inter-contact times have negligible impact on mean end-to-end delay;

- load balancing is not an effective tool for delay optimization in DTN.

The rest of the paper is organized as follows. Section 2 gives the description of the opportunistic network model, discusses the stability issues, and finds exact results for sojourn time in the source node and mobile node. Section 3 proposes and analyses an approximation for the sojourn time in the mobile queue. In Section 4, we numerically validate the accuracy of the approximation and we present additional results which give insight on the delay of the network. Section 5 concludes the paper and suggests some research directions.

\section{MODEL AND EXACT RESULTS}

\subsection{Model}

We consider a tandem model consisting of 3 first-in-firstout (FIFO) single-server systems with unlimited queue, $Q_{i}$, $i=1,2,3$, in which customers arrive to $Q_{1}$ and subsequently 
require service at $Q_{2}$ before reaching their destination at $Q_{3}$. The special feature of the model is that $Q_{2}$ alternates between positions $L_{1}$ and $L_{2}$ such that the server in $Q_{1}$ is available only when $Q_{2}$ is at $L_{1}$ and the server in $Q_{2}$ is available only when $Q_{2}$ is at $L_{2}$. In addition, $Q_{2}$ incurs a switching time from $L_{i}$ to $L_{j}(i \neq j, j \in\{1,2\})$ during which the server at neither $Q_{1}$ nor $Q_{2}$ is available. $Q_{3}$ is a sink and will not be included in our analysis.

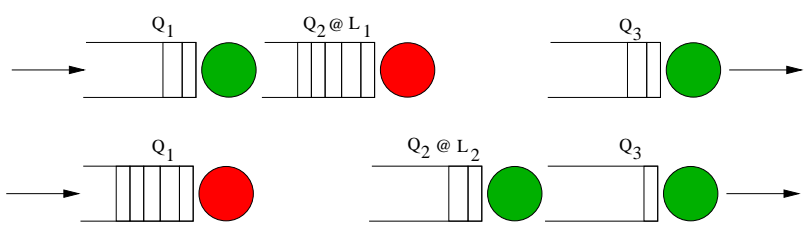

Figure 1: Three queue tandem model with a mobile queue. Top: $Q_{2}$ is at $L_{1}$, its server is down. Bottom: $Q_{2}$ at $L_{2}$, its server is up.

Customers arrive to $Q_{1}$ according to a Poisson process with arrival rate $\lambda$. The service requirement $S_{i}$ at $Q_{i}$ has general distribution $S_{i}(\cdot)$, with Laplace-Stieltjes Transform (LST) $\tilde{S}_{i}(\cdot)$, and mean $1 / \beta_{i}$. We assume that the service requirements are independent and identically distributed (iid) random variables (rvs).

Movement of $Q_{2}$ is autonomous. $Q_{2}$ remains at location $L_{1}$ (resp. $\left.L_{2}\right)$ a (random) time of duration $X_{n}^{L_{1}}$ (resp. $X_{n}^{L_{2}}$ ) before it migrates to $L_{2}$ (resp. $L_{1}$ ) during its $n$-th visit. It is important to notice that in the analysis we will use visit (time) rather than contact (time) to refer to (the duration of) a contact opportunity as to be in line with the common practice in the polling literature. After the $n$-th visit to $L_{1}, Q_{2}$ incurs a switch-over time $C_{n}^{1,2}$ from $L_{1}$ to $L_{2}$, and similarly a switch-over time $C_{n}^{2,1}$ after the $n$-th visit to $L_{2}$. We assume that $C_{n}^{1,2}\left(C_{n}^{2,1}\right)$ is an iid sequence with general distribution $C^{1,2}(\cdot)\left(C^{2,1}(\cdot)\right)$, LST $\tilde{C}^{1,2}(\cdot)\left(\tilde{C}^{2,1}(\cdot)\right)$, and mean $c^{1,2}$ $\left(c^{2,1}\right)$. We further assume that $X_{n}^{L_{1}}\left(X_{n}^{L_{2}}\right)$ is an iid sequence of common exponential distribution with rate $\alpha_{1}\left(\alpha_{2}\right)$. Furthermore, we assume $\left\{X_{n}^{L_{1}}, X_{n}^{L_{2}}, C_{n}^{1,2}, C_{n}^{2,1}\right\}$ are iid and mutually independent, and also independent at their starting time points of the other rvs in the model (queue length, waiting time, sojourn time, etc.). Therefore, the location of $Q_{2}$ is driven by an underlying continuous-time, discrete-state, process $\{L(t): t \geq 0\}$ of state space $\{-2,-1,0,1\}$. More precisely, $L(t)=1(L(t)=0)$ when $Q_{2}$ is at $L_{1}$ (resp. $\left.L_{2}\right)$ at time $t$, and $L(t)=-1(L(t)=-2)$ when $Q_{2}$ switches from $L_{1}$ to $L_{2}$ ( $L_{2}$ to $\left.L_{1}\right)$. Without loss of generality, let $L(0)=1$.

During the availability of the server at $Q_{1}$ and $Q_{2}$, the server may alternate between service and idle periods depending on whether customers are present. It is worth pointing that the term customer throughout this paper will designate packet. When the server is active at the end of a visit of $Q_{2}$ to $L_{1}$ or $L_{2}$, service will be preempted. At the beginning of the next visit of $Q_{2}$, the service time will be re-sampled according to $S_{i}(\cdot)$. This discipline is commonly referred to as preemptiverepeat-random. Let $N_{i}(t)$ denote the number of customers in $Q_{i}, i=1,2$, at time $t$. Assume $N_{i}(0)=0, i=1,2$.

A word on the notation. $\mathbf{1}_{\{A\}}$ will designate the indicator function of event $A\left(\mathbf{1}_{\{A\}}=1\right.$, if $A$ is true, and 0 otherwise), rv will mean random variable, LST Laplace-Stieltjes Trans- form and p.g.f. probability generating function. Given a rv $X, X(t)$ will denote its distribution function, $\tilde{X}(s)$ its LST.

Our objective is to analyze the sojourn time of a customer in the whole system and at the individual queues $Q_{1}$ and $Q_{2}$. First, we discuss the stability of the system. Second, we will analyze the sojourn time at $Q_{1}$. The model for $Q_{1}$ in isolation boils down to a single-vacation model or an on-off server model. Finally, we analyze the sojourn time at $Q_{2}$. To this end, we determine the joint queue-length probabilities at a specific instant. These probabilities can be related to the time-equilibrium probabilities for the tandem system. Applying Little's law, the mean sojourn time is then readily found.

\subsection{Stability condition}

The tandem model is stable if each customer in the system can be served in a finite period of time. We must consider stability on a per-queue basis as service capacity cannot be exchanged between the queues. We say that the system is stable if and only if all the queues in the system are stable.

Let a cycle define the time that separates two consecutive visits to a queue. Due to the independence assumption on our rv's, cycle lengths are iid, with generic rv $C:=$ $X^{L_{1}}+X^{L_{2}}+C^{1,2}+C^{2,1}$. For an individual queue to be stable, we must have that on average the number of customer arrivals per cycle is smaller than the number of customers that can be served at most per cycle. The latter random variable for $Q_{i}$ will be denoted by $N_{\max }^{i}, i=1,2$, and is geometrically distributed (due to the exponential visit times and preemptive-repeat-random discipline), i.e.

$$
\mathbb{P}\left(N_{\max }^{i}=k\right)=p_{i}\left(1-p_{i}\right)^{k}, k=0,1,2, \ldots,
$$

where $p_{i}=\mathbb{P}\left(\right.$ service is preempted at $\left.Q_{i}\right)=1-\tilde{S}_{i}\left(\alpha_{i}\right), i=$ 1,2 . Thus, the stability condition for $Q_{i}, i=1,2$, reads

$$
\rho_{i}:=\frac{\mathbb{E}\left[\text { arrivals per cycle to } Q_{i}\right]}{\mathbb{E}\left[N_{\text {max }}^{i}\right]}=\lambda \mathbb{E}[C] \frac{1-\tilde{S}_{i}\left(\alpha_{i}\right)}{\tilde{S}_{i}\left(\alpha_{i}\right)}<1,
$$

where

$$
\mathbb{E}[C]=\frac{1}{\alpha_{1}}+\frac{1}{\alpha_{2}}+c^{1,2}+c^{2,1} .
$$

Notice that under stability, on average, the arrival rate to $Q_{2}$ equals that to $Q_{1}$.

\subsection{Queue one}

Let us recall that we assumed that the server visit process is autonomous and that service is according to the preemptiverepeat-random discipline. It is then easily seen that $Q_{1}$ in isolation is an $\mathrm{M} / \mathrm{G} / 1$ queue with On-Off server with arrival rate $\lambda$, service time rate $\beta_{1}$, exponential on-period $X^{L_{1}}$ with rate $\alpha_{1}$, and off-period $R^{\text {off }}$ equal to the switch-over times plus the server visit time to $Q_{2}$ at $L_{2}$, i.e.,

$$
R^{o f f}=C^{1,2}+C^{2,1}+X^{L_{2}} .
$$

By a renewal reward argument, the probability, $P_{O n}$, that the server is on equals

$$
P_{O n}=\frac{1}{\alpha_{1} \mathbb{E}[C]},
$$

and $P_{O f f}=1-P_{O n}$. 
The M/G/1 queue with On-Off server has been extensively studied in the literature (see, e.g., $[7,18,19]$ ). Below, we provide an alternative derivation of the LST of the waiting time of a customer at $Q_{1}$ using the idea of waiting-time decomposition. From there, we will deduce the sojourn time and also the time-equilibrium distribution of the number of customers in $Q_{1}$. Finally, we determine the p.g.f. of the number of customers at the end of an off-period which we will need later for the analysis of $Q_{2}$.

\subsubsection{Sojourn time in queue one}

Let $A_{k}$ denote the $k$-th arriving customer at $Q_{1}$. Define the effective service time, $S_{k}^{e f f}$, of $A_{k}$ to be the time period which starts when $A_{k}$ receives service for the first time at $Q_{1}$ and which ends when $A_{k}$ departs from $Q_{1}$, i.e.,

$$
S_{k}^{e f f}=S_{k}^{*}+\sum_{i=1}^{M} C_{i},
$$

where $S_{k}^{*}$ is the conditional service requirement $S_{k}$ of $A_{k}$ given that $S_{k}$ is not interrupted, i.e., $S_{k}<X^{L_{1}}, C_{i}$ is a cycle duration distributed as $C$, and $M$ is the (random) number of off-periods before service completion of $A_{k}$. Since the visit time of $Q_{2}$ at $L_{1}, X^{L_{1}}$, is exponential and independent of the service requirement, the distribution of $M$ is geometric with parameter $p_{1}=P\left(S_{k}>X^{L_{1}}\right)=1-\tilde{S}_{1}\left(\alpha_{1}\right)$. Note that $\left\{C_{n}^{1,2}, C_{n}^{2,1}, X_{n}^{L_{1}}, X_{n}^{L_{2}}\right\}_{n>0}$ are mutually independent. In particular, the lengths of the on-periods are mutually independent and also independent of the lengths of off-periods. Further, the service discipline is preemptive-repeat-random, so that $S_{k}^{*}$ is independent of $M$ and $C_{i}, i=1, \ldots, M$. As a consequence, we can write the LST of the effective service time as follows.

$$
\tilde{S}_{k}^{e f f}(s)=\frac{\left(\alpha_{1}+s\right) \cdot \tilde{S}_{1}\left(\alpha_{1}+s\right)}{\left(\alpha_{1}+s\right)-\alpha_{1}\left(1-\tilde{S}_{1}\left(\alpha_{1}+s\right)\right) \cdot \frac{\alpha_{2} \tilde{C}^{1,2}(s) \tilde{C}^{2,1}(s)}{\alpha_{2}+s}},
$$

where $\operatorname{Re}(s) \geq 0$.

Let $W_{M / G / 1}$ denote the waiting time in the $M / G / 1$ queue with arrival rate $\lambda$ and service time $S_{k}^{\text {eff }}$. The PollaczekKhinchine formula for the LST of $W_{M / G / 1}$ reads [30, P. 386],

$$
\tilde{W}_{M / G / 1}(s)=\frac{\left(1+\lambda\left(\tilde{S}_{k}^{e f f}\right)^{\prime}(0)\right) s}{s-\lambda+\lambda \tilde{S}_{k}^{e f f}(s)},
$$

where $\left(\tilde{S}_{k}^{e f f}\right)^{\prime}(0)$ is the first derivative of $\tilde{S}_{k}^{\text {eff }}(s)$ at the origin.

The time that the service of an arriving customer to $Q_{1}$, say $A_{k}$, starts depends on the state of the system at that time. If $A_{k}$ arrives to a non-empty $Q_{1}$, then its service starts at the instant that $A_{k-1}, k \geq 1$, departs from the queue. If $A_{k}$ arrives to an empty $Q_{1}$ and the server is on, then its service starts instantaneously. However, if $A_{k}$ arrives to an empty $Q_{1}$ and the server is off, then it has to wait a residual off time, $R_{e}^{o f f}$, before its service will start. Thus, $Q_{1}$ can be seen as an On-Off queue with an exceptional first service time. It is known that for such a queue the waiting time, $W_{1}$, of a customer in $Q_{1}$ can be decomposed as follows [18, $19]$

$$
W_{1}=W_{M / G / 1}+R_{e}^{o f f} \mathbf{1}_{\{\text {server off }\}},
$$

where $W_{M / G / 1}$ and $R_{e}^{o f f}$ are independent. The LST of $W_{1}$ is readily found by conditioning on the server's state upon a customer's arrival, which yields

$$
\tilde{W}_{1}(s)=\tilde{W}_{M / G / 1}(s)\left(P_{O n}+P_{O f f} \tilde{R}_{e}^{o f f}(s)\right),
$$

where $P_{O n}$ and $\tilde{W}_{M / G / 1}(s)$ are given in (4) and (7) respectively, and

$$
\tilde{R}_{e}^{o f f}(s)=\frac{1-\left(\alpha_{2} /\left(\alpha_{2}+s\right)\right) \cdot \tilde{C}^{1,2}(s) \cdot \tilde{C}^{2,1}(s)}{\left(c^{1,2}+c^{2,1}+1 / \alpha_{2}\right) \cdot s} .
$$

The LST of the sojourn time, $D_{1} \triangleq W_{1}+S_{k}^{\text {eff }}$, of $A_{k}$ at $Q_{1}$ then yields

$$
\tilde{D}_{1}(s)=\tilde{W}_{1}(s) \tilde{S}_{k}^{e f f}(s)
$$

\subsubsection{Number of customers in $Q_{1}$}

The arrival process to $Q_{1}$ is Poisson with rate $\lambda$. Thus, it follows that the p.g.f. of $N_{1}$, which we denote by $F_{1}(\cdot)$, can be expressed as function of $\tilde{D}_{1}(\cdot)$ using the so-called functional form of Little's law (see [31] for a general proof for FIFO queues with non-anticipating arrivals) as follows

$$
F_{1}(z)=\tilde{D}_{1}(\lambda(1-z)), \quad|z| \leq 1 .
$$

Next, we will determine $F^{\{-2,1\}}(\cdot)$, the p.g.f. of the number of customers at the end of an off-period, i.e., at the transition of $L(t)$ from -2 to 1 . This function will be required later in the analysis for the second queue. To this end, we first compute the p.g.f. of the number of customers in $Q_{1}$ given that the server is on. Let $T_{o}$ denote a random time during an off-period, $T_{s}$ denote the start time of this off-period, and let $A(x, y)$ denote the total number of arrivals during $(x, y]$ with $y>x$. Thus, $N_{1}\left(T_{o}\right)=N_{1}\left(T_{s}\right)+A\left(T_{s}, T_{o}\right)$. Conditioned on the event 'server on', we may treat the epochs at which the server switches off (and immediately on) as a Poisson arrival stream of batches. Due to the PASTA property, these batches see time average behavior upon arrival. As the system observed by the arriving batches is exactly the system as observed by the server that departs, we have that

$$
\begin{aligned}
E\left[z^{N_{1}} \mid \text { server off }\right] & =E\left[z^{N_{1}\left(T_{s}\right)}\right] E\left[z^{\Psi}\right] \\
& =E\left[z^{N_{1}} \text { |server on }\right] E\left[z^{\Psi}\right],
\end{aligned}
$$

where $\Psi$ is the number of arrivals to $Q_{1}$ during the age of the off-period. The latter being equal in distribution to the residual time of an off-period. In other words, $\Psi$ is the number of Poisson arrivals of rate $\lambda$ during $R_{e}^{o f f}$. Thus, conditioned on the state of $Q_{1}$ 's server at a random time, the conditional p.g.f. of $N_{1}$ can be written as

$$
\begin{aligned}
E\left[z^{N_{1}} \mid \text { server on }\right] & =\frac{F_{1}(z)}{P_{O n}+E\left[z^{\Psi}\right] P_{O f f}} \\
& =\tilde{W}_{M / G / 1}(\lambda(1-z)) \tilde{S}_{k}^{e f f}(\lambda(1-z)) .
\end{aligned}
$$

Finally, we can conclude that

$$
F^{\{-2,1\}}(z)=E\left[z^{N_{1}} \mid \text { server on }\right] \tilde{R}^{\text {off }}(\lambda(1-z)) .
$$

\subsection{Queues in tandem}

\subsubsection{Joint queue-length probabilities at the end of a} server visit 
In this section, we will determine the queue-length distribution at the end of a server visit at each queue of the tandem of two queues. The analysis builds on the work of Eisenberg [9] and involves setting up an iterative scheme. This iterative approach was introduced by Leung [21] for the study of a probabilistically-limited polling model. Later, this model was extended in [22] to a time-limited polling model and in [6] for a time-limited model in which the server remains at a queue even if it becomes empty. A key role in the iterative scheme is played by the (auxiliary) p.g.f.'s $\phi_{k}(\mathbf{z})$ and $\phi_{k}^{s}(\mathbf{z})$ for $\mathbf{z}:=\left(z_{1}, z_{2}\right)$, which will be explained below. In the final step of the iteration scheme $\gamma_{i}(\mathbf{z})$, the p.g.f. of the queue-length distribution at the end of a server visit to $Q_{i}$, is obtained as a function of $\phi_{k}^{s}(\mathbf{z})$.

We consider a tagged queue $i$ and we will leave out the subscript and superscript $i$ whenever it does not lead to ambiguity. Let us introduce the concept of a service period. We let a service period be a segment of a visit time such that all service periods together form exactly a visit time. The first service period of a visit starts when the server arrives to the queue. This period ends with either an interruption (due to the departure of the server) or a service completion, whichever occurs first. In the latter case, a second service period will start and this process continues until finally an interruption occurs. Each service period, except for the final service period of a visit, comprises exactly one successfully completed service. Further notice that there need not always be customers present at the start of a service period.

Let us denote by $N_{k}^{i}$ the number of customers at the end of the $k$ th service period at $Q_{i}$ and by $\kappa_{i}$ the number of service periods of a visit time of $Q_{i}$. We may then define for $k \geq 1$

$$
\phi_{k}^{i}(\mathbf{z}):=\mathbb{E}\left[z^{N_{k}^{i}} \mathbf{1}_{\left\{\kappa_{i}>k\right\}}\right] .
$$

That is, $\phi_{k}^{i}(\mathbf{z})$ is the p.g.f. of the number of customers at the queues at the end of the $k$ th service period at $Q_{i}$ and service period $k$ is not the final service period (i.e., service period $k$ ends with a successful service completion, and service period $k+1$ will occur). Similarly, we define for $k \geq 1$

$$
\phi_{k}^{s, i}(\mathbf{z}):=\mathbb{E}\left[z^{N_{k}^{i}} \mathbf{1}_{\left\{\kappa_{i}=k\right\}}\right]
$$

That is, $\phi_{k}^{s, i}(\mathbf{z})$ is the p.g.f. of the number of customers at the queues at the end of the $k$ th service period at $Q_{i}$ and $k$ is the final service period (i.e., service period $k$ will be interrupted, and service period $k+1$ will not occur). Finally, we denote by $\phi_{0}^{i}(\mathbf{z})$ the p.g.f. of the number of customers present at the beginning of a visit to $Q_{i}$. Let $N(T)$ the number of arrivals during a random period $T, I_{1}$ the (exponential) interarrival time of customers at $Q_{1}$, and $C^{i, j}(\mathbf{z})$ be the p.g.f. of the number of arrivals during a switch-over time from $Q_{i}$ to $Q_{j}$. Then, by analogy with the results of De Haan et al. [6] for a time-limited polling system, $\phi_{k}^{i}(\mathbf{z})$ and $\phi_{k}^{s, i}(\mathbf{z}), i=1,2, k=$
$1,2, \ldots$, are recursively given by

$$
\begin{aligned}
\phi_{k}^{1}(\mathbf{z}) & =\left.\phi_{k-1}(\mathbf{z})\right|_{z_{1}=0} \cdot\left(\mathbb{E}\left[\mathbf{z}^{N\left(I_{1}\right)} \mathbf{1}_{\left\{X^{\left.L_{1}>I_{1}\right\}}\right.}\right]\right. \\
& \left.\times \mathbb{E}\left[\mathbf{z}^{N\left(S_{1}\right)} \mathbf{1}_{\left\{X^{L_{1}}>S_{1}\right\}}\right] \cdot z_{2}\right)+\left(\phi_{k-1}(\mathbf{z})\right. \\
& \left.-\left.\phi_{k-1}(\mathbf{z})\right|_{z_{1}=0}\right) \cdot \mathbb{E}\left[\mathbf{z}^{N\left(S_{1}\right)} \mathbf{1}_{\left\{X^{\left.L_{1}>S_{1}\right\}}\right.}\right] \cdot \frac{z_{2}}{z_{1}}, \quad(18) \\
\phi_{k}^{2}(\mathbf{z}) & =\left(\phi_{k-1}(\mathbf{z})-\left.\phi_{k-1}(\mathbf{z})\right|_{z_{2}=0}\right) \cdot \frac{\mathbb{E}\left[\mathbf{z}^{N\left(S_{2}\right)} \mathbf{1}_{\left\{X^{L_{2}}>S_{2}\right\}}\right]}{z_{2}},
\end{aligned}
$$

and

$$
\begin{aligned}
\phi_{k}^{s, 1}(\mathbf{z}) & =\left.\phi_{k-1}(\mathbf{z})\right|_{z_{1}=0} \cdot\left(\mathbb{E}\left[\mathbf{z}^{N\left(X^{L_{1}}\right)} \mathbf{1}_{\left\{X^{L_{1}}<I_{1}\right\}}\right]\right. \\
& \left.+z_{1} \mathbb{E}\left[\mathbf{z}^{N\left(I_{1}\right)} \mathbf{1}_{\left\{X^{L_{1}}>I_{1}\right\}}\right] \mathbb{E}\left[\mathbf{z}^{N\left(X^{L_{1}}\right)} \mathbf{1}_{\left\{X^{\left.L_{1}<S_{1}\right\}}\right.}\right]\right) \\
& +\left(\phi_{k-1}(\mathbf{z})-\left.\phi_{k-1}(\mathbf{z})\right|_{z_{1}=0}\right) \\
& \times \mathbb{E}\left[\mathbf{z}^{N\left(X^{L_{1}}\right)} \mathbf{1}_{\left\{X^{\left.L_{1}<S_{1}\right\}}\right.}\right] \\
\phi_{k}^{s, 2}(\mathbf{z}) & =\left.\phi_{k-1}(\mathbf{z})\right|_{z_{2}=0} \cdot \mathbb{E}\left[\mathbf{z}^{N\left(X^{\left.L_{2}\right)}\right]}\right]\left(\phi_{k-1}(\mathbf{z})\right. \\
& \left.-\left.\phi_{k-1}(\mathbf{z})\right|_{z_{2}=0}\right) \cdot \mathbb{E}\left[\mathbf{z}^{N\left(X^{L_{2}}\right)} \mathbf{1}_{\left\{X^{\left.L_{2}<S_{2}\right\}}\right.}\right]
\end{aligned}
$$

where

$$
\begin{aligned}
& \phi_{0}^{i}(\mathbf{z})=\gamma^{3-i}(\mathbf{z}) C^{3-i, i}(\mathbf{z}), \\
& \mathbb{E}\left[\mathbf{z}^{N\left(I_{1}\right)} \mathbf{1}_{\left\{X^{\left.L_{1}>I_{1}\right\}}\right.}\right]=\frac{\lambda}{\lambda+\alpha_{1}}, \\
& \mathbb{E}\left[\mathbf{z}^{N\left(S_{i}\right)} \mathbf{1}_{\left\{X^{L_{i}}>S_{i}\right\}}\right]=\tilde{S}_{i}\left(\alpha_{i}+\lambda\left(1-z_{1}\right)\right), \\
& \mathbb{E}\left[\mathbf{z}^{N\left(X^{L_{1}}\right)} \mathbf{1}_{\left\{X^{\left.L_{1}<I_{1}\right\}}\right.}\right]=\frac{\alpha_{1}}{\lambda+\alpha_{1}}, \\
& \mathbb{E}\left[\mathbf{z}^{N\left(X^{L_{i}}\right)} \mathbf{1}_{\left\{X^{\left.L_{i}<S_{i}\right\}}\right]}=\alpha_{i} \cdot \frac{1-\tilde{S}_{i}\left(\alpha_{i}+\lambda\left(1-z_{1}\right)\right)}{\alpha_{i}+\lambda\left(1-z_{1}\right)},\right. \\
& \mathbb{E}\left[\mathbf{z}^{N\left(X^{\left.L_{2}\right)}\right]}=\frac{\alpha_{2}}{\alpha_{2}+\lambda\left(1-z_{1}\right)} .\right.
\end{aligned}
$$

Equation (18) can be explained by the following observations. First, the length of the $k$ th service period (and thus also the number of arriving customers) depends on whether at least one customer was present at the end of the previous service period. This explains why the equation consists of two parts. Second, the number of customers at all queues at the end of a service period is equal to the number present at the end of the previous service period plus the ones that arrived during the present service period. Equation (19) consists only of one part due to the fact that once $Q_{2}$ is empty no customers will be served anymore during that visit. Along the same lines as Eq. (18), Eqs. (20) and (21) are derived where it should be noticed that the number of arrivals depends on whether a service period is interrupted or not. Finally, we note that $\phi_{0}^{i}(1)=1$, while $\phi_{k}^{i}(1) \leq 1$, for all $k=1,2, \ldots$, since the $k$ th period completion may not occur at all during a visit to $Q_{i}$.

Notice that there is one-to-one relationship between a visit completion and the end of a final service period. Therefore, we can write for the number of customers at the queues at the end of a server visit to $Q_{i}$

$$
\gamma^{i}(\mathbf{z})=\mathbb{E}\left[z^{N_{\kappa_{i}}^{i}}\right]=\sum_{k=1}^{\infty} \mathbb{E}\left[z^{N_{k}^{i}} \mathbf{1}_{\left\{\kappa_{i}=k\right\}}\right]=\sum_{k=1}^{\infty} \phi_{k}^{s, i}(\mathbf{z}) .
$$


We set up an iterative scheme to obtain $\gamma^{i}(\mathbf{z})$ numerically. The scheme is constructed in terms of Discrete Fourier Transforms (DFTs) as these appear more convenient for computational purposes. To this end, we replace $z_{i}, \forall i$, in the expressions above by $\omega_{i}^{k_{i}}$, where $\omega_{i}=\exp \left(-2 \pi I / J_{i}\right)$, so that all expressions become functions of $\mathbf{k}=\left(k_{1}, k_{2}\right)$. Here $I$ is the imaginary unit and $J_{i}$ refers to the number of discrete points used for $Q_{i}$ to determine the joint probabilities. These probabilities that will eventually follow are exact for $J_{i} \rightarrow \infty, \forall i$. However, the strength of the approach is that in general the probabilities are already close to the exact probabilities for small values of $J_{i}$. The pseudo-code of the iterative scheme is presented in Table 1. Notice that we start initially with an empty system. The standard values for the convergence parameters that have been used are $\epsilon=10^{-6}$ and $\delta=10^{-9}$. Finally, via the Inverse Fourier Transform, the joint queue-length probabilities at visit completion instants $\gamma_{\mathbf{n}}^{i}$ are found. These probabilities are only exact for $J_{i} \rightarrow \infty, i=1,2$ but the strength of the approach is that in general the probabilities are already close to the exact values for small values of $J_{i}$. However, it should be noted that when the system load increases, these values $J_{i}$ must typically be increased to guarantee the accurate computation of the probabilities. Thus, this iterative approach appears mainly applicable to systems with a light to moderate load.

\begin{tabular}{|c|}
\hline$\gamma^{i_{0}}(\mathbf{k})=1, \forall i_{0}, \forall \mathbf{k} ;$ (start with an empty system) \\
\hline FOR $i_{1}=1,2$ \\
\hline set $i_{2}:=i_{1} ;$ \\
\hline REPEAT \\
\hline$\hat{\gamma}^{i_{2}}(\mathbf{k})=\gamma^{i_{2}}(\mathbf{k}), \forall \mathbf{k} ;$ \\
\hline set $j:=0 ;$ \\
\hline set $\phi_{0}(\mathbf{k})=\gamma^{3-i_{2}}(\mathbf{k}) \cdot C^{3-i_{2}, i_{2}}(\mathbf{k}) ;$ \\
\hline REPEAT \\
\hline set $j:=j+1 ;$ \\
\hline $\operatorname{compute} \phi_{j}^{i_{2}}(\mathbf{k}), \forall \mathbf{k}$, using $(18)$ and $(19) ;$ \\
\hline $\operatorname{compute} \phi_{j}^{s, i_{2}}(\mathbf{k}), \forall \mathbf{k}$, using $(20)$ and $(21) ;$ \\
\hline $\operatorname{compute} \gamma^{i_{2}}(\mathbf{k})=\sum_{l=1}^{j} \phi_{l}^{s, i_{2}}(\mathbf{k}), \forall \mathbf{k} ;$ \\
\hline UNTIL $1-\operatorname{Re}\left(\gamma^{i_{2}}(\mathbf{0})\right)<\delta$ \\
\hline set $i_{2}:=\operatorname{MOD}\left(i_{2}, 2\right)+1 ;$ \\
\hline UNTIL $\left|\operatorname{Re}\left(\gamma^{i_{1}}(\mathbf{k})\right)-\operatorname{Re}\left(\hat{\gamma}^{i_{1}}(\mathbf{k})\right)\right|<\epsilon, \forall \mathbf{k}$ \\
\hline END $($ FOR $)$ \\
\hline
\end{tabular}

Table 1: Pseudo-code of iterative scheme for determining $\gamma^{i}(\mathbf{k}), \forall i$.

\subsubsection{Mean sojourn time}

The sojourn time is related to the time-equilibrium queuelength probabilities. These probabilities can be obtained from the queue-length probabilities at visit completion instants due to exponential visit times. We determine these probabilities by conditioning on the position of the server. Notice that the server is either at some queue or switching from one queue to another. Using the same arguments as in Sect. 2.3.2 above Eq. (13), we have that a departing server observes the system in steady-state conditioned on the queue it departs from. Let us further denote the p.g.f. of the number of customers present at a random instant during a switch-over time from $Q_{3-i}$ to $Q_{i}$ by $C_{R}^{i}(\mathbf{z})$. It can readily be found that

$$
C_{R}^{i}(\mathbf{z})=\gamma^{i}(\mathbf{z}) \cdot \frac{1-\tilde{C}^{3-i, i}\left(\lambda\left(1-z_{1}\right)\right)}{c^{3-i, i} \cdot \lambda\left(1-z_{1}\right)} .
$$

Hence, by conditioning on the position of the server, we may write for $P(\mathbf{z}):=\mathbb{E}\left[z_{1}^{N_{1}} z_{2}^{N_{2}}\right]$, the joint p.g.f. of the time-equilibrium queue lengths,

$$
P(\mathbf{z})=\frac{1}{\mathbb{E}[C]} \sum_{i=1}^{2}\left(\gamma^{i}(\mathbf{z}) \cdot \frac{1}{\alpha_{i}}+C_{R}^{i}(\mathbf{z}) \cdot c^{3-i, i}\right) .
$$

The mean queue length at $Q_{i}, \mathbb{E}\left[N_{i}\right]$, is then given by

$$
\mathbb{E}\left[N_{i}\right]=\sum_{n_{1} \geq 0} \sum_{n_{2} \geq 0} \mathbb{P}\left(N_{1}=n_{1}, N_{2}=n_{2}\right) n_{i},
$$

where the probabilities $\mathbb{P}\left(N_{1}=n_{1}, N_{2}=n_{2}\right)$ follow immediately from $P(\mathbf{z})$. The mean sojourn time is related to the queue length via Little's law, which then finally provides us with

$$
\mathbb{E}\left[D_{i}\right]=\mathbb{E}\left[N_{i}\right] / \lambda
$$

REMARK 1. We note that using the distributional form of Little's law also higher moments can be obtained for the end-to-end sojourn time. However, this form cannot be applied to the individual sojourn time at $Q_{2}$ here, since the arrival process to $Q_{2}$ does not satisfy the non-anticipating property [31].

\section{APPROXIMATION}

In this section, we present an approximation for $\tilde{D}_{2}(s)$, the LST of the sojourn time of a customer in the mobile queue $Q_{2}$, so that we may also deal with the situations in which the exact approach is no longer computationally feasible. We consider the workload process in $Q_{2}$ when $L(t)=0$, i.e. $Q_{2}$ is served. This will be done under the additional assumption that the service time requirements are exponentially distributed at both queues. It turns out that this process corresponds to the workload process in an $M / M / 1$ with batch arrivals. The sojourn time of a customer in $Q_{2}$ then equals the sum of the waiting time of the batch in this corresponding $M / M / 1$ system, the service times of all customers in its batch served up to and including this customer and the time the customer is at $Q_{2}$ but $L(t) \neq 0$, i.e. $Q_{2}$ is not served. We emphasize that in this case both the preemptive-repeat-random and preemptive-resume disciplines are stochastically identical. For the sake of simplicity, in the following we will consider the preemptive-resume discipline.

\subsection{The workload in queue two}

To study the workload process at $Q_{2}$, we split the time in disjoint intervals which begin at the time instants that the $L(t)$-process jumps from state -2 to 1 (i.e., the start of an on-period at $\left.Q_{1}\right)$. Denote the starting points of these intervals by $\left\{Z_{n}, n=1,2, \cdots\right\}$, with, by convention, $Z_{1}=0$. Let the $n$-th cycle of $L(t)$ denote the time interval $\left[Z_{n}, Z_{n+1}[\right.$. The duration of the $n$-th cycle is $Z_{n+1}-Z_{n}=X_{n}^{L_{1}}+C_{n}^{1,2}+$ $X_{n}^{L_{2}}+C_{n}^{2,1}$, where $X_{n}^{L_{1}}$ is the duration of time that customers can arrive at $\left.Q_{2}(L(t)=1)\right)$ and $X_{n}^{L_{2}}$ is the duration of time that customers can leave $\left.Q_{2}(L(t)=0)\right)$ during this $n$-th cycle. Let $V(t)$ denote the workload (i.e., virtual waiting time) of $Q_{2}$ present at time $t$. Without loss of generality, we assume that $V(t)$ is left-continuous, i.e., arrivals are not counted as being in the system until (just) after they arrive. A sample path of the evolution of $V(t)$ as function of $L(t)$ is shown in Figure 2. 


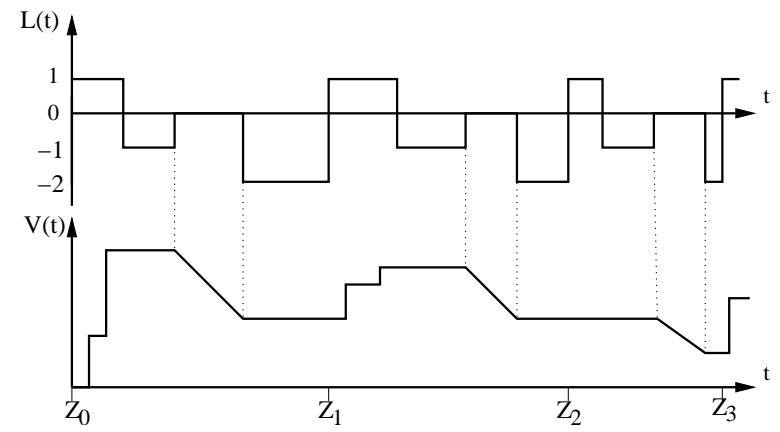

Figure 2: Evolution of $L(t)$ and workload $V(t)$ of queue $Q_{2}$.

Let $W_{n}^{B}$ denote the workload present in $Q_{2}$ at time $Z_{n}$. Based on the evolution of $L(t)$, it is easily seen that

$$
W_{n+1}^{B}=\left(W_{n}^{B}+\sum_{i=1}^{K_{n}} S_{2, i}-X_{n}^{L_{2}}\right)^{+}, \quad n \geq 0,
$$

where $(.)^{+}=\max (., 0), K_{n}$ is the total number of arrivals to $Q_{2}$ (or departures from $Q_{1}$ ) during $X_{n}^{L_{1}}$ and $S_{2, i}$ is the service requirement of a customer in $Q_{2}$. Note that $S_{2, i}$ is independent of $X_{n}^{L_{2}}$ and that $K_{n}$ depends on $N_{1}\left(Z_{n}\right)$, the number of customers at $Q_{1}$ at time $Z_{n}$. Therefore, the rvs $K_{n}, n=1,2, \cdots$ are correlated. For the sake of model tractability it is assumed in the sequel that $K_{n}, n=1,2, \cdots$ are iid and also independent of $\left\{X_{m}^{L_{2}}: m \leq n\right\}$. By these assumptions, Eq. (27) also represents the relation between the workload seen by the first customer of the $n$-th batch and of the $(n+1)$-th batch in a queue with Poisson batch arrivals with rate $\alpha_{2}$, independent batch size $K_{n}$, and exponential service requirement with rate $\beta_{2}$. It is well known that this queue is stable when

$$
-\alpha_{2} G^{\prime}(0)=\frac{\alpha_{2}}{\beta_{2}} \mathbb{E}\left[K_{n}\right]<1
$$

Note that this condition (28) is equivalent to the condition in (1) for $Q_{2}$. Furthermore, the LST of the steady-state distribution of $W_{n}^{B}$ can be written as

$$
\tilde{W}^{B}(s)=\left(1+\alpha_{2} G^{\prime}(0)\right) \frac{s}{s-\alpha_{2}+\alpha_{2} G(s)},
$$

where $G(s):=E\left[e^{-s \sum_{i=1}^{K_{n}} S_{2, i}}\right]$. By conditioning on $K_{n}$, we find that

$$
G(s)=\mathbb{E}\left[\left(\frac{\beta_{2}}{\beta_{2}+s}\right)^{K_{n}}\right]
$$

Finally, let $\tilde{V}^{j}(s)$ denote the LST of the workload seen by the $j$ th customer within a batch upon arrival including the work brought in by himself. Since the service requirement of customers is independent of the workload present in the queue upon arrival and its distribution is exponential with rate $\beta_{2}, \tilde{V}^{j}(s)$ reads

$$
\tilde{V}^{j}(s)=\tilde{V}^{j-1}(s) \frac{\beta_{2}}{\beta_{2}+s}, \quad j=1,2, \ldots,
$$

with $\tilde{V}^{0}(s)=\tilde{W}^{B}(s)$. Moreover, since $K_{n}$ are iid rvs, $\mathbb{P}(J=j)$, the probability that a customer is the $j$-th customer within the batch is equal to the fraction of customers who are $j$-th arrival in their own batch, which gives

$$
\mathbb{P}(J=j)=\frac{\mathbb{P}\left(K_{n} \geq j\right)}{\mathbb{E}\left[K_{n}\right]} .
$$

Removing the condition on the customer position in a batch, the LST of the sojourn time of an arbitrary customer in the batch arrival queue is given by

$$
\tilde{V}(s)=\beta_{2} \tilde{W}^{B}(s) \frac{1-G(s)}{s \mathbb{E}\left[K_{n}\right]} .
$$

Thus, it remains to compute $\mathbb{E}\left[z^{K_{n}}\right]$ in order to find $\tilde{W}_{B}(s)$. In the following section, we will compute the closed form of $\mathbb{E}\left[z^{K_{n}}\right]$ by using the matrix-geometric approach.

\subsection{The p.g.f. of the batch size distribution}

As remarked in the previous section, $K_{n}$ is the total number of departures from $Q_{1}$ during the $n$-th cycle and depends on the queue length of $Q_{1}$ at time $Z_{n}$. To compute the p.g.f. of $K_{n}$, we first assume that $Q_{1}$ has a limited queue of $M-1$ customers. This queue is denoted by $Q_{1}^{M}$. Later, we will let $M$ tend to infinity to get the final results.

As we need the arriving batch size distribution in steady state, we assume that $Q_{1}^{M}$ is in steady state at time $Z_{n}$. The probability that there are $i$ customers in $Q_{1}^{M}$ at $Z_{n}$ is denoted by $b_{M}(i)$. Under the assumption that the unlimited $Q_{1}$ is stable, $\lim _{M \rightarrow \infty} b_{M}(i)=b(i)$ with $\sum_{i \geq 0} b(i) z^{i}=$ $F^{\{-2,1\}}(z)$ (see Eq. $\left.(15)\right)$. Let $b_{M}=\left(b_{M}(0), \cdots, b_{M}(M-1)\right)$ denote the steady-state distribution of the finite capacity $Q_{1}^{M}$.

Let $\left(N_{1}(t), D(t)\right)$ denote the two dimensional, continuous time process with discrete state space $\{0,1, \cdots, M-1\} \times$ $\{0,1, \cdots\} \cup\{(M, 0)\}$, where $N_{1}(t)$ represents the number of customers in $Q_{1}$ at time $t$, and $D(t)$ the number of departures from $Q_{1}$ until $t$. $(M, 0)$ is an absorbing state. We refer to this absorbing Markov chain by AMC. The absorption of AMC occurs when the server leaves the queue which happens with rate $\alpha_{1}$. By setting the probability that the initial state of $\mathbf{A M C}$ at $t=0$ is $(i, 0)$ to $b_{M}(i)$, the probability that the absorption of AMC occurs from one of the states $\{(i, k): i=0,1, \cdots, M-1\}$ equals the steady-state batch size distribution $\mathbb{P}\left(K_{n}=k\right)$. The transition state diagram of AMC is shown in Figure 3.

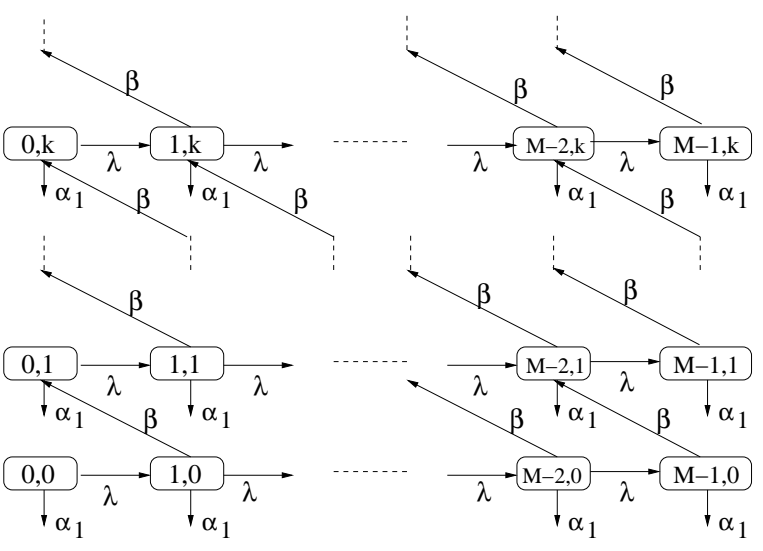

Figure 3: Transition state diagram of AMC. 
Now we focus on $\mathbb{P}\left(K_{n}=k\right)$. From Figure 3 , we readily seen that the transition matrix $\mathbf{P}$ of $\mathbf{A M C}$ can be written as

$$
\mathbf{P}=\left(\begin{array}{c|c}
\mathbf{Q} & \mathbf{R} \\
\hline \mathbf{0} & 0
\end{array}\right)
$$

where $\mathbf{Q}$ is an upper bidiagonal square block matrix, $\mathbf{R}=$ $\left(\alpha_{1}, \ldots, \alpha_{1}\right)^{T}$, and $\mathbf{0}$ is the row vector with all zero entries. The blocks of $\mathbf{Q}$ 's diagonal are all equal to $\mathbf{A}$, the $M$-by$M$ bidiagonal matrix $\mathbf{A}$ with diagonal $\left(-\lambda-\alpha_{1},-\lambda-\alpha_{1}-\right.$ $\beta_{1}, \ldots,-\lambda-\alpha_{1}-\beta_{1},-\alpha_{1}-\beta_{1}$ ) and with upper-diagonal $(\lambda, \ldots, \lambda)$. The blocks of $\mathbf{Q}$ 's upper-diagonal are all equal to $\mathbf{B}$, the $M$-by- $M$ lower-diagonal matrix with lower-diagonal $\left(\beta_{1}, \ldots, \beta_{1}\right)$.

In the sequel, $\mathbb{P}\left(K_{n}=k\right)$ is derived as function of the inverse of $\mathbf{Q}$, that is readily obtained as

$$
\mathbf{Q}^{-1}=\left(\begin{array}{ccccc}
\mathbf{A}^{-1} & \mathbf{U}_{0,1} & & \cdots & \\
& \ddots & \ddots & & \\
& & \mathbf{A}^{-1} & \mathbf{U}_{m, m+1} & \cdots \\
& & & \ddots & \ddots
\end{array}\right)
$$

where $\mathbf{U}_{m, l}=\left(-\mathbf{A}^{-1} \mathbf{B}\right)^{l-m} \mathbf{A}^{-1}$ for $m \geq 0$ and $l \geq$ $m$. Note that the matrix $\mathbf{A}$ is invertible since it is upperbidiagonal with strictly negative diagonal entries.

From the theory of absorbing Markov chains, given that the initial state vector of $\mathbf{A M C}$ is $b_{M}$, the probability that the absorption occurs at one of the states $\{(i, k): i=0,1, \cdots$, $M-1\}$ is given by (see, e.g., [11], [12, Theorem 11.9])

$$
\mathbb{P}\left(K_{n}=k\right)=-\alpha_{1} b_{M}\left(\mathbf{U}_{0, k}\right) e=-\alpha_{1} b_{M}\left(-\mathbf{A}^{-1} \mathbf{B}\right)^{k} \mathbf{A}^{-1} e .
$$

where $e$ denote the $M$-dimensional column vector with all entries equal to one. Thus, the LST of $K_{n}$ reads

$$
E_{M}\left[z^{K_{n}}\right]=-\alpha_{1} b_{M}(\mathbf{A}+z \mathbf{B})^{-1} e,
$$

where $|z| \leq 1$. Therefore, it remains to find $(\mathbf{A}+z \mathbf{B})^{-1}$.

Now, define $\mathbf{Q}(z):=(\mathbf{A}+z \mathbf{B})$, let $u^{T}=(1,0, \ldots, 0)$ and let $v^{T}=(0, \ldots, 0,1)$. Observe that $\mathbf{Q}(z)=\mathbf{T}(z)+\beta_{1} u u^{T}+$ $\lambda v v^{T}$, where $\mathbf{T}(z)$ is the M-by-M tridiagonal Toeplitz matrix with diagonal entries equal to $\left(-\lambda-\beta_{1}-\alpha_{1}\right)$, upper-diagonal entries equal to $\lambda$, and lower-diagonal entries $z \beta_{1}$. Let $t_{i j}^{*}$ denote the $(i, j)$-entry of $\mathbf{T}^{-1}(z)$. By applying the ShermanMorrison formula [24, page 76$]$ we find that the $(i, j)$-entry of $\mathbf{Q}^{-1}(z)$ gives

$$
q_{i j}^{*}=m_{i j}-\lambda \frac{m_{i M} m_{M j}}{1+\lambda m_{M M}},
$$

with

$$
m_{i j}=t_{i j}^{*}-\beta_{1} \frac{t_{i 1}^{*} t_{1 j}^{*}}{1+\beta_{1} t_{11}^{*}},
$$

for $i=1, \ldots, M$ and $j=1, \ldots, M$.

The inverse of a tridiagonal Toeplitz matrix has been computed in closed-form (see [8, Sec. 3.1]). Following that same approach, we obtain

$$
t_{i j}^{*}=\left\{\begin{array}{cl}
-\frac{\left(r_{1}^{i}-r_{2}^{i}\right)\left(r_{1}^{M+1-j}-r_{2}^{M+1-j}\right)}{\lambda\left(r_{1}-r_{2}\right)\left(r_{1}^{M+1}-r_{2}^{M+1}\right)} & , i \leq j \leq M \\
\frac{\left(r_{1}^{-j}-r_{2}^{-j}\right)\left(r_{1}^{M+1} r_{2}^{i}-r_{2}^{M+1} r_{1}^{i}\right)}{\lambda\left(r_{1}-r_{2}\right)\left(r_{1}^{M+1}-r_{2}^{M+1}\right)} & , j \leq i \leq M
\end{array}\right\}
$$

where

$$
r_{1,2}=\frac{\left(\lambda+\beta_{1}+\alpha_{1}\right) \mp \sqrt{\left(\lambda+\beta_{1}+\alpha_{1}\right)^{2}-4 \lambda \beta_{1} z}}{2 \lambda} .
$$

We take $\left|r_{1}\right|<\left|r_{2}\right|$. Note that $\left|r_{1}\right|<1<\left|r_{2}\right|$.

Inserting (36)-(37) into (35) yields that

$$
\begin{aligned}
E_{M}\left[z^{K_{n}}\right]= & -\alpha_{1} \sum_{i=1}^{M} b_{M}(i-1) \sum_{j=1}^{M}\left[t_{i j}^{*}-\frac{\beta_{1} t_{i 1}^{*} t_{1 j}^{*}}{1+\beta_{1} t_{11}^{*}}\right. \\
& \left.-\frac{\lambda m_{i M}}{1+\lambda m_{M M}}\left(t_{M j}-\frac{\beta_{1} t_{M 1}^{*} t_{1 j}^{*}}{1+\beta_{1} t_{11}^{*}}\right)\right] .
\end{aligned}
$$

Thus, it remains to let $M \rightarrow \infty$ in (40) in order to find $\mathbb{E}\left[z^{K_{n}}\right]$. It is readily seen that

$$
\begin{aligned}
\lim _{M \rightarrow \infty} t_{M, M-j} & =-\frac{1}{\lambda r_{2}} \lim _{M \rightarrow \infty} r_{1}^{j}, \\
\lim _{M \rightarrow \infty} m_{M-i, M} & =\lim _{M \rightarrow \infty} t_{M-i, M}=-\frac{1}{\lambda} \lim _{M \rightarrow \infty} r_{2}^{-(i+1)}, \\
\lim _{M \rightarrow \infty} t_{1 j} & =-\frac{1}{\lambda} r_{2}^{-j}, \\
\lim _{M \rightarrow \infty} t_{i 1} & =\frac{1}{\lambda r_{1} r_{2}} \lim _{M \rightarrow \infty} \frac{r_{1}^{M+1}}{r_{2}^{M-i+1}}-r_{1}^{i} .
\end{aligned}
$$

Some easy but technical calculus shows that the following limit is equal to zero

$\lim _{M \rightarrow \infty} \alpha_{1} \sum_{i=1}^{M} b_{M}(i-1) \sum_{j=1}^{M} \frac{\lambda m_{i M}}{1+\lambda m_{M M}}\left(t_{M j}-\frac{\beta_{1} t_{M 1} t_{1 j}}{1+\beta_{1} t_{11}}\right)$,

and that

$$
\mathbb{E}\left[z^{K_{n}}\right]=\frac{\alpha_{1}}{\lambda\left(1-r_{1}\right)\left(r_{2}-1\right)}\left[1+\beta_{1} \frac{1-z}{\lambda r_{2}-\beta_{1}} F^{\{-2,1\}}\left(r_{1}\right)\right],
$$

where $F^{\{-2,1\}}($.$) and r_{1,2}$ are given in (15) and (39) respectively. Inserting $z=\beta_{2} /\left(\beta_{2}+s\right)$ into (41) gives the closed form of $G(s)$, the LST of the service requirement of a total batch (see (30)), which in turn gives the closed form of $\tilde{W}_{B}(s)$.

\subsection{Sojourn time in queue two}

In the beginning of the section, we already remarked that, $D_{2}$, the sojourn time of a customer in $Q_{2}$ consists of three parts: the waiting time and the service time of a customer in a batch arrival queue, and the time a customer is in $Q_{2}$ but $Q_{2}$ is not served. Together, the waiting time and service time in the batch arrival queue form the sojourn time of the customer in the batch arrival queue.

Let $H_{0}$ denote the sojourn time of a costumer in the batch arrival queue. Let $\left\{H_{t}: t \geq 0\right\}$ denote the remaining sojourn time of a customer in $Q_{2}$ if the server would be continuously working at $Q_{2}$ from time $t$ onwards. In other words, $H_{t}$ decreases at rate 1 when $L(t)=0$ and $H_{t}$ is constant when $L(t) \in\{-2,-1,1\}$ at time $t$. The service at $Q_{2}$ is interrupted by the mobility of the queue. Let $Y$ denote the number of service interruptions during the sojourn time of a customer. Figure 4 displays a sample path of the evolution 
of $H_{t}$ as a function of $t$, in this figure the threshold zero is crossed at $Y=3$.

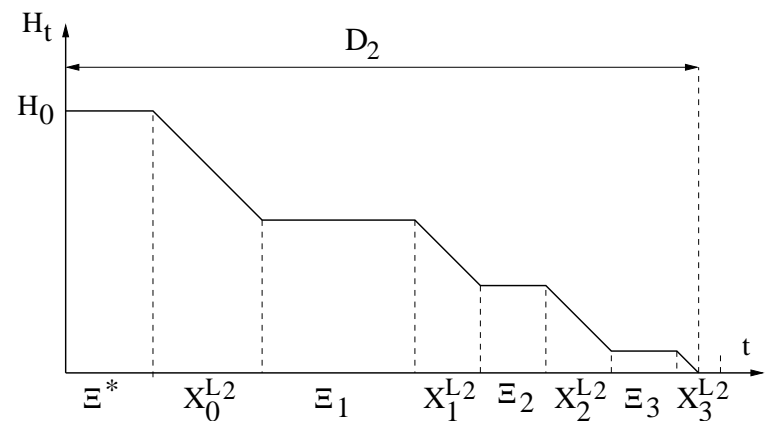

Figure 4: Evolution of $H_{t}$ as a function of $t$ with $Y=3$.

The visit periods have an exponential length with rate $\alpha_{2}$. Now, given that $H_{0}=v$, the number of interruptions has a Poisson distribution with

$$
\mathbb{E}\left[z^{Y} \mid H_{0}=v\right]=e^{-\alpha_{2} v(1-z)} .
$$

The duration of these interruptions are independent and are given by $\Xi=C^{2,1}+X^{L_{1}}+C^{1,2}$. Furthermore, $\Xi^{*}$, the time it takes before $H_{t}$ actually starts decreasing after time 0 , satisfies $\Xi^{*}=X_{e}^{L_{1}}+C^{1,2}$, where $X_{e}^{L_{1}}$ is the residual time of $X^{L_{1}}$. Note that $X_{e}^{L_{1}}$ and $X^{L_{1}}$ are identically distributed with common exponential distribution.

From Figure 4 it is easily seen that

$$
D_{2}=\Xi^{*}+H_{0}+\sum_{i=1}^{Y} \Xi_{i}
$$

By conditioning on $H_{0}$ and $Y$, we find for the LST of $D_{2}$,

$$
\begin{aligned}
\tilde{D}_{2}(s) & =\mathbb{E}\left[e^{-s \Xi^{*}}\right] \mathbb{E}\left[e^{-s\left(\sum_{i=1}^{Y} \Xi_{i}+H_{0}\right)}\right], \\
& =\mathbb{E}\left[e^{-s \Xi^{*}}\right] \mathbb{E}\left[e^{-s H_{0}} e^{-\alpha_{2} H_{0}(1-\tilde{\Xi}(s))}\right] .
\end{aligned}
$$

where $\tilde{\Xi}(s)=\frac{\alpha_{1}}{\alpha_{1}+s} \tilde{C}^{1,2}(s) \tilde{C}^{2,1}(s)$. Since $H_{0}$ equals the sojourn time in the batch arrival queue, we find (see, Eq. (33))

$$
\tilde{D}_{2}(s)=\frac{\alpha_{1} \tilde{C}^{1,2}(s)}{\alpha_{1}+s} \times \tilde{W}^{B}(\Delta(s)) \times \frac{\beta_{2}}{\mathbb{E}\left[K_{n}\right]} \times \frac{1-G(\Delta(s))}{\Delta(s)},
$$

where $\Delta(s):=s+\alpha_{2}(1-\tilde{\Xi}(s))$.

\section{NUMERICAL EVALUATION}

The evaluation of the model will be done in three parts. First, we will extensively validate the accuracy of the approximation. Second, we consider the impact of the switchover time distribution on the mean sojourn time. Notice that the switch-over times determine to a large extent the inter-contact times. Finally, we study the problem of optimizing the end-to-end delay in the network by adjusting the visit time parameters for a given cycle length. Throughout this section, the distribution of the switch-over times of $Q_{2}$, $C^{1,2}$ and $C^{2,1}$, are assumed identically distributed according to an exponential distribution with mean $c^{1,2}=c^{2,1}$.

\subsection{Model validation}

We validate the approximate model developed in Section 3.3 for the mean sojourn time at $Q_{2}$. In this model, $K_{n}$, the batch sizes in the batch arrival queue (see, e.g., (27)), were assumed to be mutually independent and independent of all other rvs. The validation will be done by comparing the results with those of the exact model in Section 2.4. We recall that due to the state-space expansion, the computation time for the exact joint queue-length probabilities, and thus also the mean sojourn time, may grow large for certain model parameters. Therefore, in the latter case we will use simulation to determine the mean sojourn time in $Q_{2}$.

Now, let us introduce some notation. Let $\mathbb{E}\left[D_{2}^{a p p}\right]$ (resp. $\mathbb{E}\left[D_{2}^{\text {exa }}\right]$ ) denote the mean sojourn time in $Q_{2}$ using the approximate (resp. exact) model given in Sect. 3.3 (resp. in Sect. 2.4.2). Let $R_{2}$ denote the absolute relative difference between the approximate and exact mean sojourn time in $Q_{2}$, i.e.,

$$
R_{2}:=\left|1-\frac{\mathbb{E}\left[D_{2}^{a p p}\right]}{\mathbb{E}\left[D_{2}^{e x a}\right]}\right| .
$$

Further, we note that the load at $Q_{1}$ and $Q_{2}$ can be written as

$$
\rho_{i}=\frac{\lambda}{\beta_{i}}\left(\frac{\alpha_{1}+\alpha_{2}}{\alpha_{3-i}}+2 \alpha_{i} c^{1,2}\right), \quad i=1,2 .
$$

Figure 5 displays $R_{2}$ as a function of $\lambda$ for different values of $c^{1,2}$ with $\beta_{1}=\beta_{2}=1$ and $\alpha_{1}=\alpha_{2}=0.1$. Thus, in this scenario the load at $Q_{1}$ and $Q_{2}$ are equal $\left(\rho_{1}=\rho_{2}\right)$. Observe that $R_{2}$ increases with $\lambda$ and that the approximate model is accurate for $\rho_{1}=\rho_{2}<0.5$. This is because the probability that $Q_{1}$ is empty upon the departure of the server from $Q_{1}$ decreases with $\lambda$. For this reason, the auto-correlation of $K_{n}$ increases with $\lambda$. Moreover, Figure 5 shows that $R_{2}$ decreases with $c^{1,2}$ for $\rho_{1}=\rho_{2}$ (e.g., for $\rho_{1}=0.5, R_{2}=15 \%$ when $c^{1,2}=1$ sec and $R_{2}=8 \%$ when $\left.c^{1,2}=20\right)$. This is because in the case where $\rho_{1}=\rho_{2}, \lambda$ decreases with $c^{1,2}$.

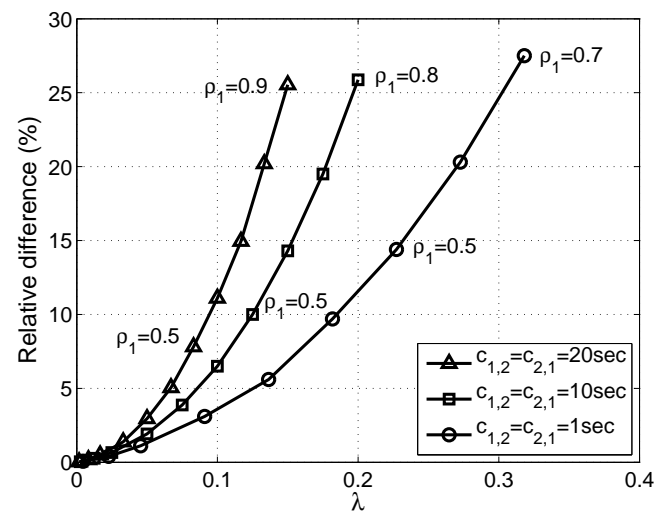

Figure 5: $R_{2}$ as a function of $\lambda$ for different values of $c^{1,2}$ with $\beta_{1}=\beta_{2}=1$ and $\alpha_{1}=\alpha_{2}=0.1$.

Figure 6 shows the mean sojourn time in $Q_{2}$ using the approximate and exact models. Observe that the approximation gives an upper bound for $\mathbb{E}\left[D_{2}\right]$. This observation is in support of the result in [3] which proves that in the correlated $M / G / 1$ a positive correlation between the service requirement and the last inter-arrival reduces the mean sojourn time. We should emphasize that also in our model $K_{n}$ 
and the last inter-arrival are positively correlated, i.e., an increase of the last interarrival time induces stochastically an increase of $K_{n}$

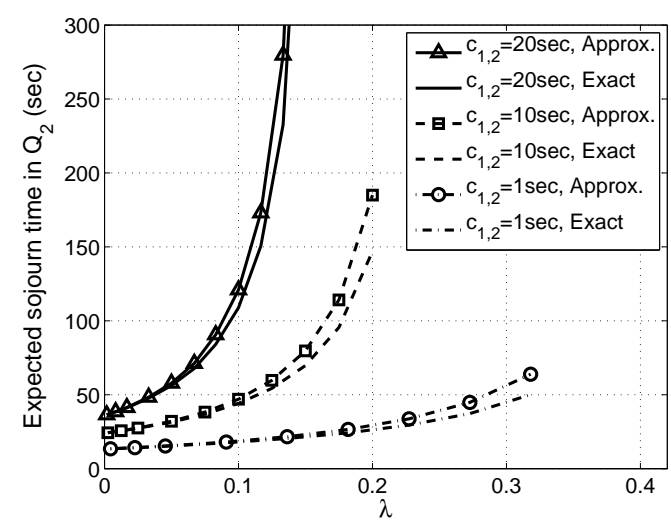

Figure 6: Mean sojourn time in $Q_{2}$ calculated from the approximate model and the exact model (resp. simulation for $\lambda>0.05$ ) as a function of $\lambda$ for different values of $c^{1,2}$ with $\beta_{1}=\beta_{2}=1$ and $\alpha_{1}=\alpha_{2}=0.1$.

Figure 7 shows $R_{2}$ as a function of $\rho_{2}$ for different values of $\rho_{1}$ with $\lambda, \beta_{1}$, and $\alpha_{1}=\alpha_{2}$ constant. This is done by changing the value of $\beta_{2}$. First, observe that for a given $\rho_{1}$, the approximate model is more accurate for small values of $\rho_{2}$. This is due to the increase of probability that $Q_{2}$ is empty at a batch arrival instant which in turn decreases the correlation between the sojourn times of customers in different batches. Second, for a given $\rho_{2}$, the approximate model is more accurate for higher values of $\rho_{1}$ (for example when $\rho_{2}=0.6, R_{2}=9.6 \%$ for $\rho_{1}=0.25$, while $R_{2}=4.72 \%$ for $\left.\rho_{1}=0.75\right)$. The reason is that for high values of $\rho_{1}$ the queue size of $Q_{1}$ is large for most of the time, therefore in this case $K_{n}$ will only depend on $X_{n}^{L_{1}}$ and $S_{1}$, the service requirement of a customer in $Q_{1}$. Since the sequences $\left\{X_{n}^{L_{1}}\right\}_{n>0}$ and $\left\{S_{1, i}\right\}_{i \geq 0}$ are independent, the auto-correlation of $\left\{K_{n}\right\}_{n \geq 0}$ becomes smaller for higher values of $\rho_{1}$.

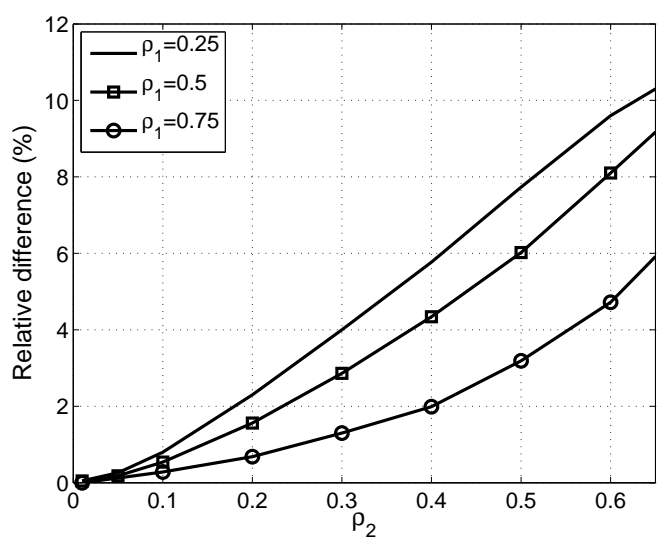

Figure 7: $R_{2}$ as a function of $\rho_{2}$ for different values of $\rho_{1}$ with $\lambda=0.01, \alpha_{1}=\alpha_{2}=0.1$, and $c^{1,2}=10$.

We conclude that the approximate model has the following properties:

- It is accurate for low and moderate load at $Q_{1}$ and $Q_{2}$;

\begin{tabular}{|l|l|l|l|l|l|l|}
\hline$S C O V_{s}$ & 1 & 5 & 10 & 15 & 20 & 30 \\
\hline$S C O V_{i}$ & 0.33 & 1.22 & 2.33 & 3.44 & 4.55 & 6.78 \\
\hline \hline & \multicolumn{7}{|c|}{ Hyper-exponential distribution } \\
\hline $\mathbb{E}\left[D^{\text {app }}\right]$ & 45.07 & 55.85 & 69.28 & 82.69 & 96.06 & 122.7 \\
\hline $\mathbb{E}\left[D^{\text {exa }}\right]$ & 45.02 & 55.8 & 69.25 & 82.66 & 95.96 & 121.4 \\
\hline \hline & \multicolumn{7}{|c|}{ Coxian distribution } \\
\hline $\mathbb{E}\left[D^{\text {app }}\right]$ & 45.07 & 55.85 & 69.31 & 82.74 & 96.15 & 122.9 \\
\hline $\mathbb{E}\left[D^{\text {exa }}\right]$ & 45.02 & 55.81 & 69.27 & 82.71 & 96.12 & 122.6 \\
\hline \hline & \multicolumn{7}{|c|}{ Weibull distribution } \\
\hline $\mathbb{E}\left[D^{\text {sim }}\right]$ & 45.01 & 55.85 & 69.25 & 82.54 & 95.88 & 121.9 \\
\hline
\end{tabular}

Table 2: Mean sojourn time in $Q_{1}$ and $Q_{2}$ as a function of $S C O V$ for the hyper-exponential, Coxian, and Weibull distributions of the switch-over times with $\lambda=0.01, \beta_{1}=\beta_{2}=1, \alpha_{1}=\alpha_{2}=0.1$, and $c^{1,2}=10$.

- It gives an upper bound for the sojourn time at $Q_{2}$;

- It is accurate for high load at $Q_{1}$ and moderate load at $Q_{2}$.

\subsection{Impact of the switch-over times distribu- tion on sojourn time}

We note that in the analysis the distribution of the switchover time was assumed to be arbitrary. This section studies the impact of the distribution of the switch-over times on the end-to-end sojourn time of a customer. This will be done by considering the following three different distributions of the switch-over times in such way that they share the same first two moments: two-phase hyper-exponential, two-phase Coxian and Weibull distribution.

For $c^{1,2}=10$, Table 2 displays the mean sojourn time as a function of $S C O V_{s}:=\operatorname{Var}\left(C^{1,2}\right) /\left(c^{1,2}\right)^{2}$, the squared coefficient of variation of the switch-over times, and of $S C O V_{i}$ $:=\operatorname{Var}\left(C^{1,2}+C^{2,1}+X^{L_{2}}\right) /\left(2 c^{1,2}+1 / \alpha_{2}\right)^{2}$, the squared coefficient of variation of the inter-visit times. This is done using both the approximate and exact models for the hyperexponential and Coxian distributions. For the Weibull distribution, we used simulation since its LST it is not known in closed form. In our simulation settings, we fixed the confidence interval to be within $1.2 \%$ of the mean simulated value. Observe that in Table 2 the mean sojourn time is almost equal for the three different distributions. Hence, we conclude that the mean sojourn time depends on the distribution of the switch-over and inter-visit times through their first two moments. In other words, considering two different distributions of the switch-over time with equal first two moments and different higher moments will induce the same mean sojourn time.

\subsection{Insight on the optimal end-to-end sojourn time}

In this section we study the evolution of, $\alpha_{2}^{o p t}$, the optimal value of $\alpha_{2}$ that yields the minimum value of the end-to-end sojourn time in $Q_{1}$ and $Q_{2}$. This will be done under the constraints of zero switch-over time, i.e., $c^{1,2}=0$, and of constant cycle length, i.e., $\mathbb{E}[C]=1 / \alpha_{1}+1 / \alpha_{2}$ is constant. Moreover, the load at $Q_{1}$ and $Q_{2}$ should be between zero and one. Note that under these constraints when $\alpha_{1}$ increases $\alpha_{2}$ should decrease. Since the mean sojourn time in $Q_{1}$ (resp. $Q_{2}$ ) increases (resp. decreases) with $\alpha_{1}$ (resp. $\alpha_{2}$ ) then 
$\alpha_{2}^{\text {opt }}$ exists and it is unique. The adjustment of $\alpha_{1}$ and $\alpha_{2}$ can be done in practice by controlling the transmission power of the nodes in our model.

In the following, $\alpha_{2}^{o p t}$ will be computed using the approximate mean sojourn time in (45) using the numerical optimization package of MAPLE. This value was validated by verifying that the mean sojourn time using the exact model for $\alpha_{2}=\alpha_{2}^{o p t}$ is a local minimum inside $\left[\alpha_{2}^{o p t}-10^{-3}, \alpha_{2}^{o p t}+\right.$ $\left.10^{-3}\right]$.

In the symmetric case $\beta_{1}=\beta_{2}$, it is found that $\alpha_{2}^{\text {opt }}=\alpha_{1}=$ $2 / \mathbb{E}[C]$. In the asymmetric case $\beta_{1}>\beta_{2}=1$, Table 3 displays $\alpha_{2}^{\text {opt }}$ as a function of $\beta_{1}$ for $\mathbb{E}[C]=10$. Observe that in this case $\alpha_{2}^{\text {opt }}$ is smaller than $2 / \mathbb{E}[C]$ and that this difference increases with $\beta_{1}$. Table 4 displays $\alpha_{2}^{o p t}$ as a function of $\beta_{2}$ for $\beta_{2}>\beta_{1}=1$ and $\mathbb{E}[C]=10$. In contrast with the previous case, notice that $\alpha_{2}^{\text {opt }}$ is greater than $2 / \mathbb{E}[C]$. In fact, the values of $\alpha_{2}^{o p t}$ and $\alpha_{1}$ for these two cases are exchanged which is quite surprising since the arrival processes at the queues are essentially different. It is not clear why these values found for $\alpha_{2}^{\text {opt }}$ would indeed lead to the optimal mean sojourn time.

\begin{tabular}{|l|l|l|l|l|l|l|}
\hline$\beta_{1}$ & 1.1 & 2 & 3 & 6 & 11 & 16 \\
\hline$\alpha_{2}^{\text {opt }}$ & 0.197 & 0.181 & 0.173 & 0.165 & 0.161 & 0.16 \\
\hline$\alpha_{1}$ & 0.203 & 0.223 & 0.236 & 0.253 & 0.265 & 0.27 \\
\hline$\rho_{1}(\%)$ & 4.62 & 2.79 & 1.97 & 1.05 & 0.6 & 0.42 \\
\hline$\rho_{2}(\%)$ & 4.91 & 4.50 & 4.34 & 4.12 & 4.01 & 3.97 \\
\hline
\end{tabular}

Table 3: $\alpha_{2}^{o p t}$ as a function of $\beta_{1}$ for $\beta_{2}=1, \lambda=0.025$, and $\mathbb{E}[C]=10$.

\begin{tabular}{|l|l|l|l|l|l|l|}
\hline$\beta_{2}$ & 1.1 & 2 & 3 & 6 & 11 & 16 \\
\hline$\alpha_{2}^{\text {opt }}$ & 0.203 & 0.223 & 0.236 & 0.253 & 0.265 & 0.27 \\
\hline$\alpha_{1}$ & 0.197 & 0.181 & 0.173 & 0.165 & 0.161 & 0.16 \\
\hline$\rho_{1}(\%)$ & 4.92 & 4.52 & 4.34 & 4.13 & 4.01 & 0.42 \\
\hline$\rho_{2}(\%)$ & 4.61 & 2.79 & 1.96 & 1.05 & 0.60 & 3.97 \\
\hline
\end{tabular}

Table 4: $\alpha_{2}^{\text {opt }}$ as a function of $\beta_{2}$ for $\beta_{1}=1, \lambda=0.025$, and $\mathbb{E}[C]=10$.

In practice one might prefer to have a simple rule that provides a value for $\alpha_{2}$ which yields a mean sojourn time close to optimal. Therefore, we will discuss two alternative, heuristic optimization approaches. First, we select the values of $\alpha_{1}$ and $\alpha_{2}$ such that the load is balanced at both queues, i.e., $\rho_{1}=\rho_{2}$. This gives:

$$
\alpha_{i}=\frac{\beta_{1}+\beta_{2}}{\beta_{3-i}} \cdot \frac{1}{\mathbb{E}[C]}, i=1,2 .
$$

Second, we choose $\alpha_{1}$ and $\alpha_{2}$ based on the analysis of a tandem model of two $\mathrm{M} / \mathrm{M} / 1$ queues with shared service capacity. That means that the servers at both queues are always present, but serving at rate $\nu$ at $Q_{1}$ and at rate $1-\nu$ at $Q_{2}$. Then, the optimal $\nu$, say $\nu^{*}$, is the one that minimizes the end-to-end sojourn in such a tandem model, which we denote by $\mathbb{E}[D]^{M / M / 1}$ and equals simply

$$
\mathbb{E}[D]^{M / M / 1}=\frac{1}{\beta_{1} \nu-\lambda}+\frac{1}{\beta_{2}(1-\nu)-\lambda} .
$$

We choose the ratio $\alpha_{1} / \alpha_{2}$ equal to $\left(1-\nu^{*}\right) / \nu^{*}$, such that the fraction the server is at $Q_{1}$ in our model equals the optimal rate $\nu^{*}$ in the $\mathrm{M} / \mathrm{M} / 1$ tandem model.
In Tables 5 and 6 , we present the results of this comparison. Here, $\alpha_{2}^{o p t}, \alpha_{2}^{L B}$ and $\alpha_{2}^{M / M / 1}$ refer to the choice of $\alpha_{2}$ in the optimal case, in the load balancing heuristic, and in the $\mathrm{M} / \mathrm{M} / 1$ tandem heuristic, respectively. Further, we present the relative differences in mean sojourn time using the two heuristics (denoted by $\epsilon^{L B}$ and $\epsilon^{M / M / 1}$ ) with respect to the optimal mean sojourn time, $\mathbb{E}[D]^{\text {opt }}$. In Table 5 , we study the performance of those heuristics when $\beta_{1}$ is increased while $\beta_{2}, \lambda$ and $\mathbb{E}[C]$ are kept constant. We note that for the symmetric case, $\beta_{1}=\beta_{2}$, the heuristics would also give the optimal solution $\alpha_{1}=\alpha_{2}$. The performance using load balancing worsens rapidly when $\beta_{1}$ is increased. Also the $\mathrm{M} / \mathrm{M} / 1$ tandem heuristic deviates from the optimum, but the relative differences remain small. In Table 6 , we investigate the performance of the heuristics when the mean cycle time is varied. The results show that the relative error when using load balancing is almost insensitive to $\mathbb{E}[C]$. We note that in the limit case of the cycle time tending to zero our tandem model approaches the tandem model of two M/M/1 queues. Hence, in this case the M/M/1 tandem heuristic is optimal. This explains the why the relative error increases in $\mathbb{E}[C]$. However, notice that the relative error $\epsilon^{M / M / 1}$ is still very small for $\mathbb{E}[C]=20$.

We can conclude that balancing the load is not a good solution for end-to-end sojourn time optimization unless $\beta_{1} \approx$ $\beta_{2}$. However, using an optimization heuristic based on a simple tandem model of two $\mathrm{M} / \mathrm{M} / 1$ queues will give nearly optimal results for the mean sojourn time under a wide variety of parameter settings.

\begin{tabular}{|l|c|c|c|c|c|c|}
\hline$\beta_{1}$ & 1.1 & 2 & 3 & 6 & 11 & 16 \\
\hline$\alpha_{2}^{\text {opt }}$ & 0.194 & 0.174 & 0.166 & 0.156 & 0.150 & 0.148 \\
\hline$\alpha_{2}^{L B}$ & 0.190 & 0.150 & 0.133 & 0.117 & 0.109 & 0.106 \\
\hline$\alpha_{2}^{M / M / 1}$ & 0.195 & 0.167 & 0.154 & 0.138 & 0.128 & 0.123 \\
\hline $\mathbb{E}[D]^{\text {opt }}$ & 14.47 & 12.82 & 12.14 & 11.42 & 11.08 & 10.94 \\
\hline$\epsilon^{L B}(\%)$ & $<0.1$ & 3.9 & 8.6 & 17.2 & 23.6 & 26.3 \\
\hline$\epsilon^{M / M / 1}(\%)$ & $<0.1$ & 0.4 & 0.9 & 2.3 & 3.8 & 4.7 \\
\hline
\end{tabular}

Table 5: Comparison of $\alpha_{2}$ and $\mathbb{E}[D]$ for different optimization approaches for $\beta_{2}=1, \lambda=0.1$, and $\mathbb{E}[C]=10$.

\begin{tabular}{|l|c|c|c|c|c|}
\hline $\mathbb{E}[C]$ & 1 & 2 & 5 & 10 & 20 \\
\hline$\alpha_{2}^{\text {opt }}$ & 1.408 & 0.719 & 0.300 & 0.156 & 0.080 \\
\hline$\alpha_{2}^{L B}$ & 1.167 & 0.583 & 0.233 & 0.117 & 0.058 \\
\hline$\alpha_{2}^{M / M / 1}$ & 1.375 & 0.687 & 0.275 & 0.137 & 0.069 \\
\hline $\mathbb{E}[D]^{\text {opt }}$ & 3.152 & 4.07 & 6.83 & 11.42 & 20.58 \\
\hline$\epsilon^{L B}(\%)$ & 17.3 & 17.1 & 17.1 & 17.2 & 17.7 \\
\hline$\epsilon^{M / M / 1}(\%)$ & 0.2 & 0.6 & 1.4 & 2.3 & 3.0 \\
\hline
\end{tabular}

Table 6: Comparison of $\alpha_{2}$ and $\mathbb{E}[D]$ for different optimization approaches for $\beta_{1}=6, \beta_{2}=1$, and $\lambda=$ 0.1 .

\section{CONCLUSIONS}

This study is part of a research effort towards developing analytical models for quantifying the end-to-end delay in a opportunistic network. We consider here a network consisting of a fixed source node, a fixed destination node, and a mobile relay node. A closed-form expression has been derived for the delay at the packet's source node. Next, an iterative approach has been developed for the joint queuelength distribution of the source and the relay node. In 
addition, a simple approximate model has been proposed for the delay analysis at the relay node. The approximate model has extensively been validated and shows excellent results. Numerical results on the mean end-to-end delay show that the inter-contact time distribution impacts this metric only through its first two moments. Moreover, load balancing is not an effective tool for delay optimization, while the $\mathrm{M} / \mathrm{M} / 1$ tandem heuristic is near optimal.

In the present work, we have focused on the delay analysis of simple opportunistic networks. As a second step towards understanding these networks, we will study in the future work the scenario where multiple relay nodes coexist in the network. In this case, the packet multi-copy routing-based proposals in DTN will help to reduce the delay. We anticipate that the exact and approximate models can be extended at least to cover the packet single-copy case with only one packet transmission at a time. For the multi-copy case, the help of certain theoretical techniques like customers resequencing and impatient customers might be required to analyze the delay.

\section{REFERENCES}

[1] Delay tolerant networking research group. Web site: http://www.dtnrg.org.

[2] C. Bettstetter, H. Hartenstein, and X. Pérez-Costa. Stochastic properties of the random waypoint mobility model. ACM/Kluwer Wireless Networks, Special Issue on Modeling and Analysis of Mobile Networks, 10(5):555-567, Sept. 2004.

[3] S. Borst, O. Boxma, and M. Combé. Collection of costumers: a correlated M/G/1 queue. Performance Evaluation, 20(1):47-59, 1992.

[4] A. Chaintreau, P. Hui, J. Crowcroft, C. Diot, R. Gass, and J. Scott. Impact of human mobility on the design of opportunistic forwarding algorithm. In Proc. of IEEE INFOCOM, Barcelona, Spain, Apr. 2006.

[5] E. G. Coffman, G. Fayolle, and I. Mitrani. Two queues with alternating service periods. In Performance '87: Proc. of the 12th IFIP WG 7.3 International Symposium on Computer Performance Modelling, Measurement and Evaluation, pages 227-239, 1988.

[6] R. de Haan, R. J. Boucherie, and J.-K. van Ommeren. A polling model with an autonomous server. Research Memorandum, University of Twente, 2007.

[7] B. Doshi. Queueing systems with vacations-a survey. Queueing Systems, 1(1):29-66, 1986.

[8] M. Dow. Explicit inverses of Toeplitz and associated matrices. ANZIAM J., 44(E):E185-E215, Jan. 2003.

[9] M. Eisenberg. Queues with periodic service and changeover times. Operation Research, 20(2):440-451, 1972.

[10] I. Frigui and A. Alfa. Analysis of a time-limited polling system. Computer Communications, 21(6):558-571, 1998.

[11] D. P. Gaver, P. A. Jacobs, and G. Latouche. Finite birth-and-death models in randomly changing environments. Advances in Applied Probability, 16:715-731, 1984.

[12] C. Grinstead and J. Snell. Introduction to Probability. American Mathematical Society, 1997.

[13] R. Groenevelt, P. Nain, and G. Koole. The message delay in mobile ad hoc networks. Performance Evaluation, 62(1-4):210-228, Oct. 2005.

[14] M. Grossglauser and D. Tse. Mobility increases the capacity of ad hoc wireless networks. ACM/IEEE Transactions on Networking, 10(4):477-486, Aug. 2002.

[15] M. Ibrahim, A. Al Hanbali, and P. Nain. Delay and resource analysis in manets in presence of throwboxes. Performance Evaluation, 64(9-12):933-947, Oct. 2007.

[16] S. Jain, K. Fall, and R. Patra. Routing in a delay tolerant networking. In Proc. of ACM Sigcomm, Aug. 2004.

[17] T. Karagiannis, J.-Y. L. Boudec, and M. Vojnovic. Power law and exponential decay of inter contact times between mobile devices. In Proc. of MOBICOM, Montréal, Québec, Canada, Sep. 2007.

[18] T. Katayama. Waiting time analysis for a queueing system with time-limited service and exponential timer. Naval Research Logistics, 48:638-651, 2001.

[19] T. Katayama. Level-crossing approach to a time-limited service system with two types of vacations. Operations Research Letters, 33(3):295-300, 2005.

[20] J. Leguay, T. Friedman, and V. Conan. Evaluating mobility pattern space routing for DTNs. In Proc. of IEEE Infocom, Barcelona, Spain, Apr. 2006.

[21] K. Leung. Cyclic-service systems with probabilistically-limited service. IEEE Journal on Selected Areas in Communications, 9(2):185-193, 1991.

[22] K. Leung. Cyclic-service systems with non-preemptive time-limited service. IEEE Transactions on Communications, 42(8):2521-2524, 1994.

[23] P. Nain, D. Towsley, B. Liu, and Z. Liu. Properties of random direction models. In Proc. of IEEE INFOCOM, Miami, FL, Mar. 2005.

[24] W. Press, B. Flannery, S. Teukolsky, and W. Vetterling. Numerical Recipes in C: The Art of Scientific Computing. Cambridge University Press, 1992.

[25] T. Small and Z. J. Haas. The shared wireless infostation model: A new ad hoc networking paradigm. In Proc. of ACM MOBIHOC, Anapolis, MD, USA, 2003.

[26] T. Small and Z. J. Haas. Resource and performance tradeoffs in delay-tolerant wireless networks. In Proc. ACM SIGCOM Workshop on Delay-Tolerant Networks, Philadelphia, PA, USA, Aug. 2005.

[27] T. Spyropoulos, K. Psounis, and C. Raghavendra. Spray and wait: An efficient routing scheme for intermittently connected mobile networks. In Proc. of ACM Sigcomm workshop on Delay Tolerant Networking, Aug. 2005.

[28] T. Spyropoulos, K. Psounis, and C. S. Raghavendra. Efficient routing in intermittently connected mobile networks: The multi-copy case. To appear in ACM/IEEE Transactions on Networking (available online), 2007.

[29] T. Spyropoulos, K. Psounis, and C. S. Raghavendra. Efficient routing in intermittently connected mobile networks: The single-copy case. To appear in ACM/IEEE Transactions on Networking (available 
online), 2007.

[30] R. Wolff. Stochastic Modeling and the Theory of Queues. Prentice Hall, 1989.

[31] M. Zazanis. A palm calculus approach to functional versions of little's law. Stochastic Processes and their Applications, 74:195-201(7), 1998.

[32] E. Zhang, G. Neglia, J. Kurose, and D. Towsley. Performance modeling of epidemic routing. Computer Networks, 51(10):2867-2891, July 2007.

[33] W. Zhao, M. Ammar, and E. Zegura. Controlling the mobility of multiple data transport ferries in a delay-tolerant network. In Proc. of IEEE INFOCOM, Miami, FL, Mar. 2005. 